\title{
Chapter 15 \\ Extracting Information on the Equation of State from Binary Neutron Stars
}

\author{
Kentaro Takami, Luciano Rezzolla and Luca Baiotti
}

\begin{abstract}
Recently Bauswein and Janka [6, 7] found that the typical frequency of a hypermassive neutron star, which is called $f_{2}$ in this paper, is a simple function of the average rest-mass density, essentially independently of the equation of state considered. While expected, this result is very important to decide the system mass from observed gravitational waves. However in their simulations, the Einstein equations were solved by assuming conformal flatness and employing a gravitational radiation-reaction scheme within a post-Newtonian framework. Besides this mathematical approximation, there is also a numerical one in the use of smoothparticle hydrodynamics code, which is well-know to be particularly dissipative and that rapidly suppresses the amplitude of the bar-mode deformation and rapidly yields to an almost axisymmetric system. Therefore we have reinvestigated the calculations in their work improving on the two approximations discussed above (i.e., conformal flatness and smooth-particle hydrodynamics) to obtain an accurate description both during the inspiral and after the merger. Then we have found another typical frequency with a clear peak, which is called $f_{\mathrm{LI}}$ in this paper. Finally we show the relations between the initial masses and the $f_{\mathrm{LI}}$ and $f_{2}$ frequencies of the gravitational waves emission from a hypermassive neutron stars.
\end{abstract}

K. Takami $(\bowtie) \cdot$ L. Rezzolla

Max-Planck-Institut für Gravitationsphysik (Albert-Einstein-Institut),

Am Mühlenberg 1, 14476 Potsdam, Germany

e-mail: kentaro.takami@aei.mpg.de

Luca Baiotti

Institute of Laser Engineering, Osaka University, Suita, Osaka 567-0086, Japan

(C) Springer International Publishing Switzerland 2016 


\subsection{Introduction}

Binary systems of compact objects, such as neutron-star binaries, neutron star-black hole binaries and binary black holes, inspiral and merge as a result of the emission of gravitational waves (GWs). They are the most promising sources of GWs in a series of advanced detectors such as LIGO [1], Virgo [2] and KAGRA [3], which will be operated within next five years. Especially binary neutron star mergers (BNSs) may be the most common source with realistic detection rate of $\sim 40 \mathrm{yr}^{-1}$ [4].

It is known that GWs from hypermassive neutron stars (HMNSs) formed after the merger have a typical peak frequency (see the right panel of Fig. 15.2), which has been recently identified as the frequency of the fundamental quadrupolar fluid mode ( $f_{2}$ mode) [5]. Recently, Bauswein and Janka [6, 7] found that the typical GW frequency of a HMNS is a simple function of the average mass density, essentially independent of the EOS considered, by performing a large number of simulations. This result is very important to deduce the system mass from the observed GWs.

However in their simulations, the Einstein equations were solved by assuming the conformal flatness condition (CFC) and employing a gravitational radiation-reaction scheme within a post-Newtonian framework. Besides this approximation in the equations, their results may be affected by the numerical technique they use to solve the equations of relativistic hydrodynamics, namely smoothed-particle hydrodynamics (SPH), whose well-known large dissipativity probably suppresses rapidly the amplitude of the bar-mode deformation and so rapidly leads to an almost axisymmetric system.

In this work, we reinvestigate their work by improving on the two approximations discussed above (i.e., CFC and SPH) to obtain an accurate description both during the inspiral and after the merger. Unless explicitly stated, we use units in which $c=G=M_{\odot}=1$.

\subsection{Methodology}

All of our calculations have been performed in full general relativity. The evolution of the spacetime is obtained by using a recently developed constraint-damping CCZ4 formalism [8] with a " $1+\log$ " slicing condition and a "Gamma-driver" shift condition. The general-relativistic hydrodynamics equations are solved using the Whisky code [9], with the Marquina flux formula and a PPM reconstruction. The grid hierarchy, with a reflection symmetry condition across the $z=0$ plane, a $\pi$-symmetry condition across the $x=0$ plane and a moving-mesh refinement, is handled by the Carpet mesh refinement driver [10], where we have used six refinement levels, the finest having a resolution of $0.15 M_{\odot} \simeq 0.221 \mathrm{~km}$. Thanks to this, we can extract accurate gravitational waveforms in the very far zone from the center of the system (typically at a radius $r_{\mathrm{ex}}=500 M_{\odot} \simeq 738 \mathrm{~km}$ ). In this work, we analyse only the $\ell=m=2$ mode of GWs, which is dominated one. 
In order to close the system of equations for the hydrodynamics, we employ a "hybrid" equation of state (EOS) based on a piecewise polytropic (PP) EOS augmented by a $\Gamma$-law EOS. That is, pressure and specific internal energy are

$$
p=p_{\mathrm{ad}}+p_{\mathrm{th}}, \quad \varepsilon=\varepsilon_{\mathrm{ad}}+\varepsilon_{\mathrm{th}},
$$

where

$$
\begin{aligned}
& p_{\mathrm{th}}=\left(\Gamma_{\mathrm{th}}-1\right) \rho \varepsilon_{\mathrm{th}}, \\
& p_{\mathrm{ad}}=K_{i} \rho^{\Gamma_{i}}\left(\rho_{i-1} \leq \rho<\rho_{i}, \quad 1 \leq i \leq n\right), \\
& \varepsilon_{\mathrm{ad}}=\varepsilon_{i}+\frac{K_{i}}{\Gamma_{i}-1} \rho^{\left(\Gamma_{i}-1\right)}, \quad K_{l+1}=K_{l} \rho_{l}^{\left(\Gamma_{l}-\Gamma_{l+1}\right)} \quad(1 \leq l \leq n-1) .
\end{aligned}
$$

The detail parameters used by this work are shown in Table 15.1.

We use quasi-equilibrium initial data of irrotational equal-mass BNSs with coordinate separation $D=45 \mathrm{~km}$, which are computed by the multi-domain spectralmethod code LORENE [12] under the assumption of a conformally at spacetime metric. This configurations undergoes $\sim 3.5$ orbits in the inspiral phase and then creates a HMNS.

\subsection{Results}

Figure 15.1 shows the spectrogram of the $\ell=m=2$ plus polarization of $\mathrm{GW}, h_{+}$. Clearly there are basically two dominating frequencies, one correspond to the orbital frequency in the inspiral phase $(t<0)$ and the other is the typical GW frequency $\left(f_{2}\right.$ mode) of the HMNS after the merger $(t>0)$. As expected, the $f_{2}$ mode undergoes a large variation in time. This behavior is rather different from the one reported in [6, 7] (compare Fig. 15.1 with Fig. 15.2 of [6]). The waveform at $20 \mathrm{Mpc}$ is shown in the left panel of Fig. 15.2. It consist of the part of the inspiral, merger, HMNS and black hole. In the right panel, we show the power spectral density (PSD) of the effective amplitude which is defined by

$$
\tilde{h}(f) \equiv \sqrt{\frac{\left|\tilde{h}_{+}(f)\right|^{2}+\left|\tilde{h}_{\times}(f)\right|^{2}}{2}}, \quad \tilde{h}_{\mathrm{A}}(f) \equiv\left\{\begin{array}{ll}
2 \int h_{\mathrm{A}}(t) e^{-i 2 \pi f t} d t & (f \geq 0) \\
0 & (f<0)
\end{array},\right.
$$

where "A" indicates two polarization modes, + or $\times$. Beside a $f_{2}$ peak, we can clearly see another peak labeled $f_{\text {LI }}$ ("LI" means "Last Inspiral") in the figure. These typical peak frequencies will be observable by advanced LIGO. 


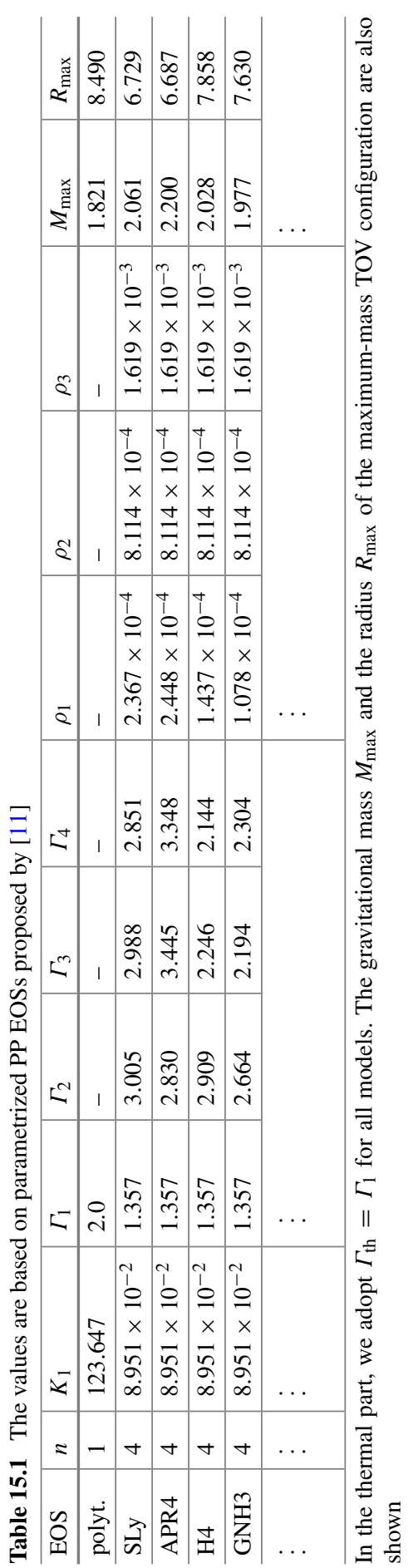




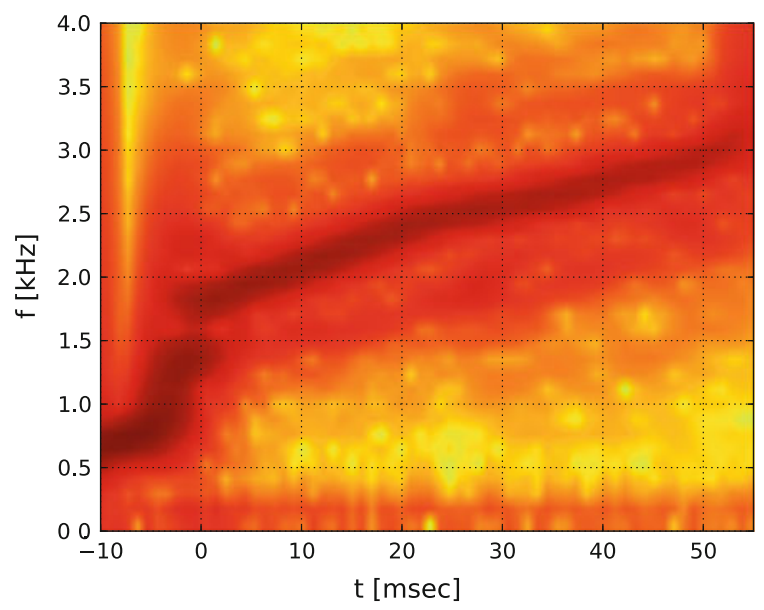

Fig. 15.1 The spectrogram of the $\ell=m=2$ plus polarization of $\mathrm{GW}, h_{+}$, for the model with $M_{\mathrm{ADM}} \approx 2.731 M_{\odot}$ and polyt. EOS, which is equivalent to two NSs with $M \approx 1.378 M_{\odot}$ at infinity. The waveform is aligned at the time of the merging [13]
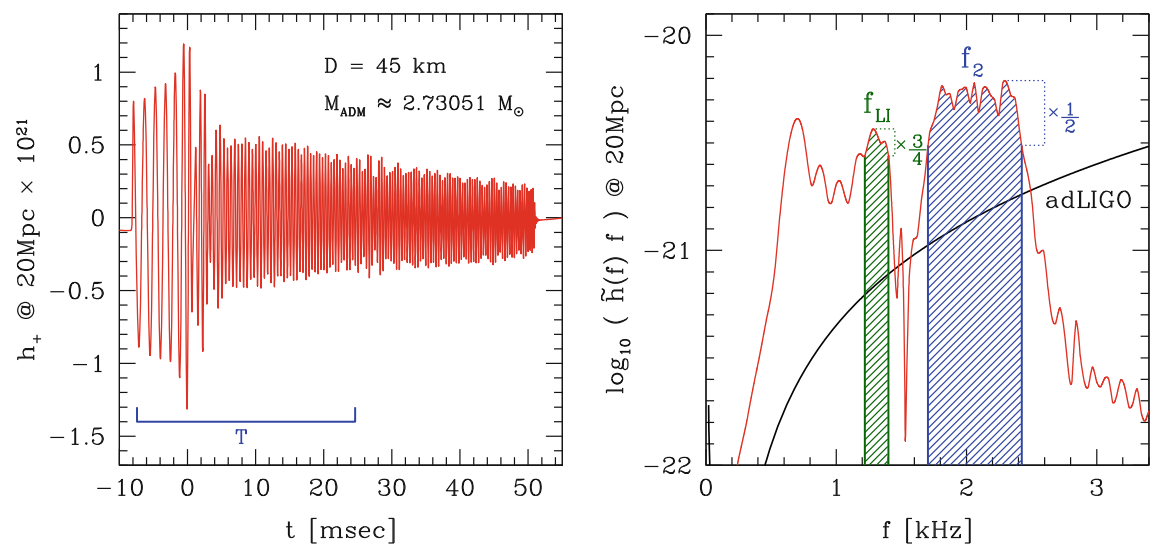

Fig. 15.2 Time evolution of the GW amplitude $h_{+}$with the phase of inspiral, merger, HMNS and BH (left) and the PSD of the effective amplitude $\tilde{h}(f) f$ in the time interval $T=$ $[-1500,5000] M_{\odot} \approx[-7.39,24.63] \mathrm{ms}$ (right) for the same model of Fig. 15.1. In the right panel, the black solid line shows the sensitivity curves, $\sqrt{S_{h}(f) f}$, of advanced LIGO [14], the green and blue shaded regions show $f_{\mathrm{L}}$ and $f_{\mathrm{R}}$, which are used for the definition of the effective peak frequencies

Because the peak frequencies change in time, we define its average as

$$
\langle f\rangle=\frac{\int_{f_{\mathrm{L}}}^{f_{\mathrm{R}}} \tilde{h}(f) f^{2} d f}{\int_{f_{\mathrm{L}}}^{f_{\mathrm{R}}} \tilde{h}(f) f d f}
$$



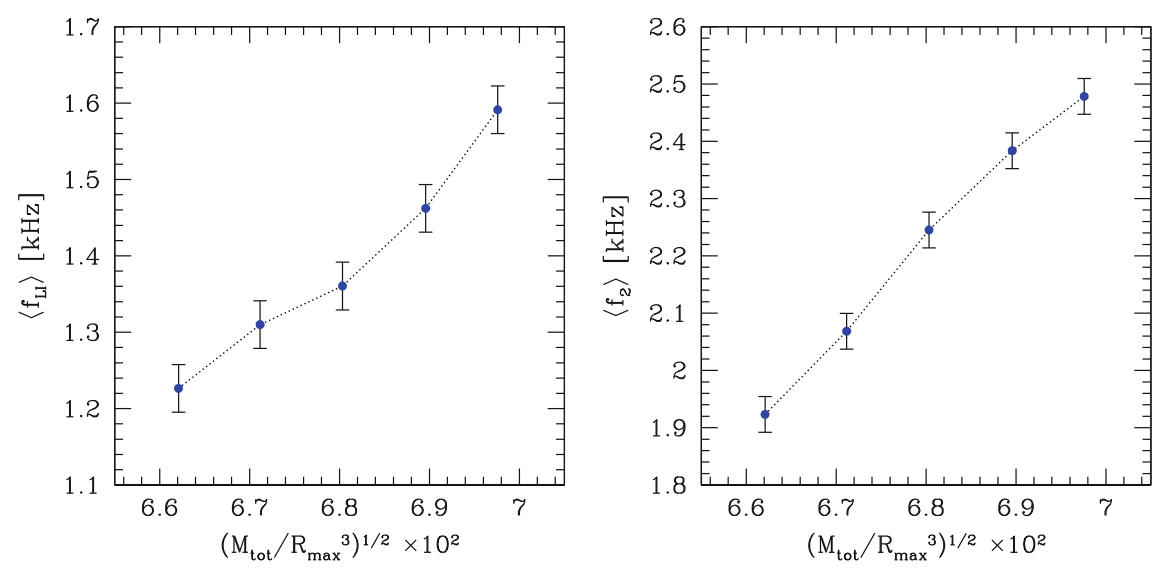

Fig. 15.3 Correlation between the square root of the specific average mass density $\sqrt{M_{\text {tot }} / R_{\max }{ }^{3}}$ and the average peak frequency of $\left\langle f_{\mathrm{LI}}\right\rangle(l e f t),\left\langle f_{2}\right\rangle$ (right)

within the time interval $T=[-1500,5000] M_{\odot} \approx[-7.39,24.63] \mathrm{ms}$, where $f_{\mathrm{L}}$ and $f_{\mathrm{R}}$ are defined by the frequencies that satisfy the condition

$$
\tilde{h}(f) f=\max \left[\tilde{h}\left(f_{\mathrm{LI}}\right) f_{\mathrm{LI}}\right] \times \frac{3}{4}, \quad \tilde{h}(f) f=\max \left[\tilde{h}\left(f_{2}\right) f_{2}\right] \times \frac{1}{2}
$$

(see the green and blue shaded region in the right panel of Fig. 15.2). In Fig. 15.3 we show the correlations between the square root of the specific average mass density $\left(M_{\mathrm{tot}} / R_{\max }{ }^{3}\right)^{1 / 2}$ and the average peak frequency of $\left\langle f_{\mathrm{LI}}\right\rangle$ and $\left\langle f_{2}\right\rangle$ in polyt. EOS, where $M_{\text {tot }}=M_{1}+M_{2}$ and $M_{1,2}$ are the gravitational masses of each NS at infinity. We can clearly see a simple linear relation for both $\left\langle f_{\mathrm{LI}}\right\rangle$ and $\left\langle f_{2}\right\rangle$. The similar correlation for $\left\langle f_{2}\right\rangle$ have been already pointed out by [6,7], while we report the correlation for $\left\langle f_{\mathrm{LI}}\right\rangle$ for the fast time. Definitely both cases have tight correlations, and these can be a powerful tool to extract the information of BNSs such as the system mass from the observed GWs.

\subsection{Final Remark}

This work is still in progress. We will provide soon complete results for several binaries obeying a variety of hybrid EOSs, although we have reported the results for only one EOS, i.e., polyt. EOS, in this paper.

Acknowledgments This work was supported in part by the DFG grant SFB/Transregio 7 and by "CompStar", a Research Networking Programme of the ESF. The simulations were performed on SuperMUC at LRZ-Munich and on Datura at AEI-Potsdam. 


\section{References}

1. G.M. Harry et al., Class. Quantum Grav. 27, 084006 (2010)

2. T. Accadia et al., Class. Quantum Grav. 28, 114002 (2011)

3. Y. Aso, Y. Michimura, K. Somiya, M. Ando, O. Miyakawa, T. Sekiguchi, D. Tatsumi, H. Yamamoto, Phys. Rev. D 88, 043007 (2013)

4. J. Abadie et al., Class. Quantum Grav. 27, 173001 (2010)

5. N. Stergioulas, A. Bauswein, K. Zagkouris, H.T. Janka, Mon. Not. R. Astron. Soc. 418, 427 (2011)

6. A. Bauswein, H.T. Janka, Phys. Rev. Lett. 108, 011101 (2012)

7. A. Bauswein, H.T. Janka, K. Hebeler, A. Schwenk, Phys. Rev. D 86, 063001 (2012)

8. D. Alic, C. Bona-Casas, C. Bona, L. Rezzolla, C. Palenzuela, Phys. Rev. D 85, 064040 (2012)

9. L. Baiotti, I. Hawke, P.J. Montero, F. Löffler, L. Rezzolla, N. Stergioulas, J.A. Font, E. Seidel, Phys. Rev. D 71, 024035 (2005)

10. E. Schnetter, S.H. Hawley, I. Hawke, Class. Quantum Grav. 21, 146588 (2004)

11. J.S. Read, D.L. Benjamin, B.J. Owen, J.L. Friedman, Phys. Rev. D 79, 124032 (2009)

12. http://www.lorene.obspm.fr

13. J.S. Read, L. Baiotti, J.D.E. Creighton, J.L. Friedman, B. Giacomazzo, K. Kyutoku, C. Markakis, L. Rezzolla, M. Shibata, K. Taniguchi, Phys. Rev. D 88, 044042 (2013)

14. B.S. Sathyaprakash, B.F. Schutz, Living reviews in relativity (2009) 